\title{
Adaptación y validación de la versión en español de la Escala de Respeto Incondicional hacia las personas en una muestra de escolares chilenos*
}

\section{Adaptation and Validation of the Spanish Version of the Scale of Unconditional Respect for Persons in Chilean Schoolchildren}

\author{
DAVID SiRLOPÚ ${ }^{\mathrm{a}}$ \\ Universidad del Desarrollo, Chile \\ ORCID: http://orcid.org/0000-0003-4683-1734 \\ Claudia Pérez-Salas \\ Universidad de Concepción, Chile \\ ORCID: http://orcid.org/0000-0002-6940-6514 \\ Verónica Villarroel \\ Universidad del Desarrollo, Chile \\ ORCID: http://orcid.org/0000-0002-3000-2248 \\ Catalina García \\ Pontificia Universidad Católica de Chile, Chile \\ ORCID: http://orcid.org/0000-0002-0698-4974 \\ KiYOMI BARRERA \\ Universidad del Desarrollo, Chile \\ ORCID: http://orcid.org/0000-0002-0032-9928 \\ Eugenio Fierro \\ Universidad del Desarrollo, Chile \\ ORCID: http://orcid.org/0000-0002-7578-7245 \\ IsIDORa CASTILlo \\ Universidad del Desarrollo, Chile \\ ORCID: http://orcid.org/0000-0002-9941-1437
}

a Autor de correspondencia. Correo electrónico: dsirlopu@udd.cl

Para citar este artículo: Sirlopú, D., Pérez-Salas, C., Villarroel, V., García, C., Barrera, K., Fierro, E., \& Castillo, I. (2019). Adaptación y validación de la versión en español de la Escala de Respeto Incondicional hacia las personas en una muestra de estudiantes chilenos. Universitas Psychologica, 18(1), 1-13. https://doi.org/10.11144/Javeriana.upsy18-1.

\section{RESUMEN}

La Escala de Respeto Incondicional hacia las Personas (ERIP) mide las actitudes positivas hacia la autonomía, la igualdad y la integridad de los seres humanos. Esta investigación validó y analizó algunas propiedades psicométricas de la versión en español de la ERIP, en una muestra de 947 escolares chilenos entre 12 y 19 años $(M=15.47, D E=1.49)$. Mediante análisis factoriales, exploratorio y confirmatorio, emergieron dos factores: Desprecio hacia los demás $(\mathrm{F} 1)$ y Respeto hacia los demás $(\mathrm{F} 2)$. La consistencia interna (F1: Omega $=0.82 ; \mathrm{F} 2$ : Omega $=0.78)$ así como la validez convergente y discriminante de la ERIP fueron adecuadas. Estos resultados indican que la ERIP es un instrumento válido y confiable para ser aplicado en adolescentes y jóvenes.

Palabras clave

respeto; validación psicométrica; análisis factorial. 


\begin{abstract}
Unconditional Respect for Persons (RfP) scale is an instrument for measuring positive attitudes towards autonomy, equality, and integrity of human beings. This research validated and analyzed some psychometric properties of the Spanish version of the RfP scale in a sample of 947 pupils from 12 and 19 years old $(\mathrm{M}=$ $15.47, S D=1.49$ ). Using exploratory and confirmatory factor analysis, we found two factors of respect Disrespect towards people (F1) and Respect toward people (F2). Internal consistency $(\mathrm{F} 1$ : Omega $=0.82 ; \mathrm{F} 2$ : Omega $=$ 0.78 ), and convergent and discriminant validity of the RfP were acceptable. Results show that RfP is a valid and reliable instrument for applying to adolescents and young population.
\end{abstract}

Keywords

respect; psychometric validation; factorial analysis.

La ubicuidad del respeto en los discursos y las prácticas cotidianas en todas las sociedades es una constatación del poderoso rol que cumple en la convivencia humana. Así, en las familias y las escuelas se les inculcan a los niños el respeto a los adultos, a las autoridades, a los símbolos patrios y a sus propios compañeros. Las relaciones sentimentales son más sólidas cuando existe un respeto mutuo dentro de la pareja. La productividad y un clima organizacional estimulante se fundan en el respeto entre jefes y empleados. Los sistemas democráticos se fortalecen cuando existe un trato respetuoso entre el partido gobernante y la oposición. Finalmente, ninguna forma de paz mundial sería posible si no se cumplieran formas y gestos que comuniquen respeto entre las naciones. Por esta razón, Lawrence-Lightfoot (2000) afirma que el respeto es "el ingrediente más poderoso en las relaciones nutritivas y en la creación de una sociedad justa" (p. 13).

\section{Aspectos generales}

El respeto es descrito como una disposición individual relativamente estable que expresa un trato considerado o deferente hacia otro (Simon, 2007). Existe cierta controversia entre los académicos sobre su naturaleza, aunque la gran mayoría de ellos se inclina a describirlo como una actitud que posee dimensiones cognitivas, motivacionales y comportamentales (Dillon, 2007). En consecuencia, el respeto se puede entender como una relación intencional que se establece entre un individuo racional y un objeto (que puede pertenecer a distintas categorías), frente al cual el individuo responde de una cierta manera, porque percibe un determinado valor inherente a dicho objeto (van Quaquebeke, Henrich, \& Eckloff, 2007). Dado que este es un concepto clave dentro de la psicología social, en esta investigación se adoptó dicho enfoque.

Existen numerosas clasificaciones de respeto, pero una de las más citadas es la del filósofo estadounidense Stephen Darwall (1977), quien distingue entre el respeto estimativo (appraisal respect) y el respeto de reconocimiento (recognition respect). El primero alude a una estimación o admiración que se le confiere a un individuo por poseer cualidades personales extraordinarias o por un comportamiento meritorio (Darwall, 2006). El objeto de este tipo de respeto es la excelencia y, por tanto, implica establecer diferencias entre categorías de grupos o personas (Simon, 2007). Desde esta perspectiva, una afirmación tal como "Roger Federer es mejor tenista que Rafael Nadal" no sería ofensiva. El respeto de reconocimiento, por su parte, es categórico, es decir, no establece distinciones según los méritos de los individuos, sino que se dispensa o no, simplemente por el mero hecho de ser personas.

En la literatura, es posible encontrar el uso de términos afines con los que el respeto está relacionado, pero que no son equivalentes. Por poner un caso, la tolerancia. Esta implica la acción de "soportar" las diferencias sobre distintos tópicos o hacia otros grupos, con los cuales se está en desacuerdo o sencillamente se los desaprueba (Lægard, 2010). Asumiendo esta definición, la tolerancia adquiere una connotación restringida y negativa, dado que un individuo solo requiere aceptar pasivamente o incluso ignorar-a la persona o grupo que le desagrada. Por el contrario, el respeto implica una expresión de agencia, que presupone el esfuerzo del individuo por comprender la situación de aquel que es distinto (Schirmer, Weidenstedt, \& Reich, 2012). 
Otra situación equivalente se puede aplicar a la confusión entre el aprecio y el respeto. Según el Modelo de Contenido de los Estereotipos (Fiske, Cuddy, Glick, \& Xu, 2002), un individuo es digno de aprecio si posee cualidades sociales muy desarrolladas tales como la calidez o la cordialidad en el trato. En cambio, el respeto solo recae en personas con elevadas competencias intelectuales o laborales. Este modelo teórico asume además que el aprecio no implica respeto, y viceversa. La distinción anterior es útil pero descuida un aspecto esencial desde un punto de vista fenomenológico: la fuente del aprecio residiría en los propios intereses o deseos del individuo, ocupando el objeto percibido un plano secundario. Por el contrario, el objeto percibido es central en el respeto por cuanto este genera que el individuo le dedique una atención especial y cuidadosa (Dillon, 2007).

\section{Psicología social y respeto}

A pesar de que una gran cantidad de interacciones sociales están reguladas e influidas por el hecho de dar y recibir respeto, solo en las dos últimas décadas los psicólogos han comenzado a estudiarlo sistemáticamente (p. ej., Haidt \& Kesebir, 2010). Uno de los primeros esfuerzos en esta línea es el Modelo del Valor del Grupo desarrollado por Tom R. Tyler que se inscribe en el marco de la justicia procedimental. Tyler et al. (Blader \& Tyler, 2009; Tyler \& Lind, 1992) han demostrado que cuando los miembros de un grupo perciben que una autoridad los ha tratado con procedimientos justos, a saber, imparciales, confiables y respetuosos, están más dispuestos a obedecer las decisiones adoptadas y a juzgarlas de forma compatible con sus valores.

Existe también un dinámico programa de investigación experimental orientado al estudio del respeto horizontal, es decir, aquel que se establece entre miembros de un estatus similar dentro de un mismo grupo (Simon, 2007). Dos son los principales modelos teóricos que predominan en este campo y que, pese a sus diferentes enfoques, ambos coinciden en que la percepción de respeto intensifica la identificación, la lealtad y el compromiso de los individuos con su grupo de pertenencia.

El primer modelo establece que la importancia del respeto reside en que, a través de este, se le transmite a los individuos que son valorados positivamente por el resto de los miembros de su grupo (De Cremer \& Mulder, 2007; De Cremer \& Tyler, 2005). El segundo, propone una explicación alternativa: el "ingrediente activo del respeto" -o lo que hace que el respeto sea esencial para los seres humanos-no se centra en la evaluación que reciben de los miembros de su grupo, sino que, a través del respeto, las personas se sienten reconocidas como un igual ante los demás (Renger, Mommert, Renger, \& Simon, 2016; Renger \& Simon, 2011).

Aunque el significado último del respeto permanece desconocido, es evidente el valor que tiene para la identidad personal y social de las personas. Ahora bien, un aspecto poco explorado en la literatura es por qué podría ser importante para un individuo sentirse respetado por un exogrupo. Este vacío teórico ha motivado la aparición de numerosas investigaciones sobre el respeto en contextos intergrupales, las cuales han ido aportando valiosos hallazgos (véanse Simon, Mommert, \& Renger, 2015). Así, encontramos estudios que han analizado el respeto subgrupal en sociedades compuestas por numerosos grupos étnicos (Huo \& Molina, 2006) o la percepción de respeto por parte de distintas minorías tales como inmigrantes (Licata, Sánchez-Masas, \& Green, 2011), homosexuales (Simon \& Grabow, 2014) o musulmanes (Blackwood, Hopkins, \& Reicher, 2013).

\section{Escala de Respeto Incondicional hacia las Personas}

Una de las limitaciones que presenta el estudio del respeto dentro de la psicología social es la escasa cantidad de instrumentos disponibles para su medición, aunque una excepción es el trabajo de Leary, Brennan y Briggs (2005). A pesar de que es posible ubicar investigaciones que analizan las consecuencias psicológicas de no ser tratado respetuosamente (Janoff-Bulman \& 
Werther, 2008), son menos frecuentes aquellas que indaguen en las actitudes generales asociadas a demostrar respeto hacia las personas. En ese sentido, la creación de la Escala de Respeto Incondicional a las Personas (en adelante, ERIP) es una contribución importante que cubre este vacío.

La ERIP fue diseñada por Lalljee, Laham y Tam (2007) con el objetivo de evaluar el respeto que se debe a cualquier persona y cuya presencia denotaría una actitud positiva hacia la autonomía, la igualdad y la integridad de los seres humanos. Sus fundamentos teóricos descansan en los principios de la ética kantiana, y puede resumirse en la famosa sentencia: "Obra de tal modo que trates a la humanidad, tanto en tu persona como en la persona de cualquier otro, siempre al mismo tiempo como un fin y nunca simplemente como medio" (Kant, 1785/1995, p. 4:429).

Originalmente, la ERIP tuvo 30 ítems que fueron aplicados a una muestra de 900 participantes adultos en el Golfo Pérsico, India y Reino Unido. A partir de los análisis estadísticos realizados, se redujeron a 12 (ocho de ellos en sentido inverso). Posteriormente, la aplicación de análisis factorial exploratorio y análisis factorial confirmatorio revelaron una solución unidimensional. Asimismo, con el objetivo de evaluar la red nomológica de la versión de 12 ítems, Lalljee et al. (2007) compararon la ERIP con constructos semejantes y opuestos al respeto incondicional. Los resultados mostraron que la ERIP correlacionó de modo directo y significativo con la "agradabilidad" (uno de los factores del modelo de los Cinco Grandes), la empatía y la toma de perspectiva y de modo inverso y significativo con la orientación a la dominancia social y la personalidad autoritaria.

La ERIP también ha sido empleada en contextos intergrupales. Por ejemplo, Lalljee, Tam, Hewstone, Laham y Lee (2009) diseñaron dos estudios con el fin de indagar en el poder predictivo del respeto incondicional sobre las tendencias a actuar a favor del endogrupo y en contra del exogrupo. El primer estudio se realizó en Dubai, donde participaron profesionales árabes, británicos e indios y el segundo se realizó en la India donde participaron individuos de distintos grupos religiosos (p. ej., hindús, cristianos, musulmanes, etc.). Por medio de análisis de regresión lineal múltiple, estos autores encontraron que el respeto fue un predictor sobresaliente en ambos estudios, reduciendo especialmente las tendencias a actuar negativamente contra el exogrupo. Este resultado se mantuvo incluso cuando otras variables (p. ej., dominancia social, empatía y contacto) fueron controladas estadísticamente.

Con el objetivo de profundizar en estos hallazgos, Laham, Tam, Lalljee, Hewstone y Voci (2010) evaluaron algunos mediadores del respeto incondicional. Utilizando modelos de ecuaciones estructurales, estos autores encontraron que los efectos del respeto incondicional sobre las tendencias a la acción intergrupal estuvieron mediados por emociones intergrupales (positivas y negativas), así como por la inclusión del otro en el sí mismo. Adicionalmente, el respeto incondicional también ha sido estudiado como variable moderadora. En Irlanda del Norte, Lalljee et al. (2007) entrevistaron a universitarios protestantes o católicos con el objetivo de analizar las reacciones de personas con altos y bajos niveles de respeto frente a situaciones intergrupales amenazantes. Los participantes con bajos niveles de respeto en condiciones de alta amenaza reportaron mayor tendencia a actuar negativamente contra el exogrupo, mientras que aquellos con altos niveles, mostraron menor tendencia a actuar negativamente contra el exogrupo, independientemente del nivel de amenaza percibido.

\section{La presente investigación}

Considerando el reducido número de instrumentos de medición del respeto existente en la literatura internacional, esta investigación propone adaptar y validar una versión en español de la ERIP en una muestra de escolares chilenos. Para lograr esto, (1) se evaluará su estructura factorial como evidencia de validez de constructo, (2) se evaluará su confiabilidad por consistencia interna según las dimensiones 
emergentes y (3) se comparará con otros constructos similares u opuestos como evidencia de validez convergente y divergente. La elección de participantes que se ubican en las etapas de la adolescencia y de la juventud temprana representa, a nuestro juicio, una excelente oportunidad para pesquisar la efectividad de los hogares y los colegios, en el fomento del respeto a las diferencias individuales y culturales, lo cual es una base necesaria para el compromiso y la defensa de los derechos humanos.

\section{Método}

Participaron del estudio 947 escolares (474 mujeres y 440 hombres; 33 personas no consignaron su sexo), cuyos rangos de edades estuvieron entre 12 y 19 años $(M=15.47, D E$ $=1.49$ ). Se realizó un muestreo no aleatorio intencional que comprendió el último año de educación básica en Chile que es octavo básico (14.7\%), primero medio (23.2\%), segundo medio (21.4\%), tercero medio (20.2 \%) y cuarto medio (17.1\%) que es el último año de escolaridad en Chile. Se debe mencionar que los alumnos de octavo básico tienen entre 12 y 14 años y los alumnos de cuarto medio, entre 17 y 19 años en promedio.

\section{Instrumentos}

Se usó una escala de respuesta tipo Likert de cinco puntos para mostrar el grado de acuerdo o desacuerdo ante distintas afirmaciones (muy en desacuerdo $=1 ;$ muy de acuerdo $=5$ ), excepto en la medida de matonaje donde también se utilizó una escala de cinco puntos, pero para determinar la frecuencia de realización de una determinada conducta (nunca $=1$; siempre $=5$ ). Para todos los instrumentos, el puntaje más alto representa la mayor presencia del atributo evaluado.

\section{Escala de Respeto Incondicional hacia las Personas}

La ERIP tiene 12 ítems, cuatro de los cuales están redactados en un sentido opuesto al foco central de la escala (Tabla 1). El procedimiento de adaptación al español aparece consignado en la sección de procedimientos.

\section{Escala de Orientación a la Dominancia Social}

Se empleó la nueva versión de ocho ítems (Ho et al., 2015) que incorpora cuatro subdimensiones de dos ítems cada una: Dominancia ("Algunos grupos de personas son simplemente inferiores a otros grupos"), Contra la dominancia ("Ningún grupo debiera dominar en la sociedad"), Antiigualitarismo ("Es injusto intentar que haya igualdad entre todos los grupos") y Contra el anti-igualitarismo ("Debiéramos hacer todo lo posible por igualar las condiciones de diferentes grupos"). Ho et al. (2015) encontraron valores de confiabilidad altos para la escala completa $(\alpha$ de Cronbach entre 0.89 y 0.95), así como para las cuatro subdimensiones ( $\alpha$ de Cronbach entre 0.79 y 0.88$)$.

\section{Escala de Toma de Perspectiva}

Se ocupó una subescala del Interpersonal Reactivity Index de Davis (1983), validado al español por Mestre, Frías y Samper (2004). Esta escala consta de ocho ítems ("Pienso que hay dos posturas en un tema e intento tener en cuenta ambas partes"; "Antes de criticar a alguien intento imaginar cómo me sentiría si estuviera en su lugar") y en el estudio de estos investigadores, dicha subescala obtuvo una confiabilidad baja $(\alpha$ $=0.56$ ).

\section{Escala de Conducta de Matonaje}

Se adaptaron nueve ítems del instrumento creado por Espelage y Holt (2001) para evaluar la conducta de bullying (matonaje), a través de diferentes conductas negativas tales como poner apodos, sembrar rumores o excluir a las personas (p. ej., "He molestado a otros/as alumnos/as"; "He ayudado a acosar a otros/as alumnos/as"). Estos autores reportaron una confiabilidad alta de esta escala $(\alpha=0.86)$. 


\section{Escala de Convicción Moral}

Se adaptó la medida elaborada por van Zomeren, Postmes y Spears (2012) para evaluar cuán fuertes son las actitudes hacia ciertos temas morales que son adoptados como principios personales. Esta escala cuenta con cinco ítems ("La superación de la condición actual de todas las minorías en Chile refleja una parte importante de lo que soy"; "La superación de la condición actual de todas las minorías en Chile es un asunto moral para mí”) y en el estudio de Zomeren et al., obtuvo una confiabilidad alta $(\alpha=0.88)$.

\section{Procedimiento}

Para la adaptación y validación al español de la ERIP, se siguieron las recomendaciones de Muñiz, Elosua y Hambleton (2013). Primero, se pidió el consentimiento del autor principal de la escala para traducir y usar su instrumento. Segundo, se hicieron adecuaciones idiomáticas del inglés al español con dos traductores independientes. Tercero, las traducciones fueron revisadas por tres psicólogos, quienes sugirieron cambiar algunas palabras o modificar frases que estaban bien traducidas pero que podrían tener dificultades de comprensión en la cultura de habla española y en el grupo etario elegido. La versión en español definitiva fue enviada a otro traductor para que la transcribiera literalmente al inglés. Al comparar la versión original en inglés con la retrotraducida, no se observaron mayores diferencias.

Con respecto al trabajo de campo, los investigadores se contactaron telefónicamente con los directivos de los colegios previamente seleccionados, con el fin de informarles de las características del estudio. Aquellos directores que aceptaron participar, debieron completar una autorización institucional. Luego, los encuestadores informaron a los alumnos de los cursos elegidos del objetivo general del estudio, y se les preguntó acerca de su interés en participar. Los escolares que voluntariamente quisieron responder el cuestionario, debieron traer un consentimiento firmado por al menos uno de sus padres o apoderados. Solo los escolares que llevaron firmados los consentimientos el día de la aplicación, pudieron participar del estudio. En retribución a su tiempo, se le entregó a cada estudiante una entrada al cine (aproximadamente 4 USD).

\section{Resultados}

Esta sección presenta el siguiente orden: (1) análisis descriptivos de la ERIP y de las otras escalas; (2) análisis factorial exploratorio (AFE) de la ERIP; (3) análisis factorial confirmatorio (AFC) para evaluar la validez de constructo y constatar la dimensionalidad de la ERIP; (4) confiabilidad de la ERIP mediante el coeficiente Omega y (5) validez convergente y divergente de la ERIP por medio de análisis de correlación con diferentes variables. Los análisis (1) y (5) fueron realizados con el programa IBM SPSS Statistics v. 19, los análisis (2) y (3) con el programa Mplus v. 7.4 y el análisis (4) con el software libre JASP v. 9 (JASP Team, 2018).

\section{Análisis descriptivos}

En la Tabla 1 se observan los estadísticos descriptivos básicos para los 12 ítems de la ERIP. Los cuatro ítems enunciados en sentido positivo obtuvieron puntajes ligeramente superiores al punto medio de la escala (3), siendo el ítem 12 el que obtuvo un mayor grado de acuerdo. En cuanto a los ítems con un sentido contrario al respeto, la mayoría de participantes obtuvo puntajes inferiores al punto medio de la escala y solo el ítem 2 se ubicó en el punto medio de la escala. En cuanto a la asimetría y la curtosis, todos los ítems estuvieron dentro del rango de -1 y +1 inferior al criterio máximo permitido por algunos autores (Gravetter \& Wallnau, 2014). 


\section{Tabla 1}

Promedios, desviación estándar, asimetría, curtosis y valores perdidos de los ítems de la ERIP

\begin{tabular}{|c|c|c|c|c|}
\hline Ítems & $M(D E)$ & Asimetría & Curtosis & $\begin{array}{l}\text { Valores } \\
\text { perdidos }\end{array}$ \\
\hline $\begin{array}{l}\text { Considerar los deseos de los demás es un aspecto } \\
\text { importante de las relaciones humanas. (ftem 1) }\end{array}$ & $3.77(1.04)$ & -0.69 & 0.14 & 9 \\
\hline $\begin{array}{l}\text { Quien ha cometido un delito terrible ya no tiene derecho a } \\
\text { ser tratado con dignidad. (Ítem } 2 \text {; inverso) }\end{array}$ & $3.02(1.15)$ & -0.05 & -0.56 & 11 \\
\hline $\begin{array}{l}\text { Miro con desprecio las debilidades y deficiencias de los } \\
\text { demás. (Item 3; inverso) }\end{array}$ & $2.17(1.18)$ & 0.78 & -0.27 & 16 \\
\hline $\begin{array}{l}\text { Las personas que son tontas merecen nuestro desprecio. } \\
\text { (Item 4; inverso) }\end{array}$ & $1.97(1.22)$ & 1.02 & -0.11 & 11 \\
\hline $\begin{array}{l}\text { Mostrar las defíciencias de una persona ante los demás es } \\
\text { necesario si es que esta no ha estado a la altura. (Ítem } 5 \text {; } \\
\text { inverso) }\end{array}$ & (1.23) & 0.65 & -0.62 & 15 \\
\hline $\begin{array}{l}\text { A veces es necesario causar mucho dolor al interrogar a } \\
\text { alguien sospechoso de haber cometido un delito terrible. } \\
\text { (ftem } 6 \text {; inverso) }\end{array}$ & $2.54(1.24)$ & 0.38 & -0.77 & 7 \\
\hline $\begin{array}{l}\text { No creo necesario ser tolerante con las personas que me } \\
\text { desagradan. (item } 7 \text {; inverso) }\end{array}$ & $2.67(1.23)$ & .23 & -0.79 & 9 \\
\hline $\begin{array}{l}\text { Tratar a todas las personas con respeto es una parte } \\
\text { esencial de nuestras relaciones con los demás. (ítem 8) }\end{array}$ & 3.93 & -0.79 & -0.07 & 11 \\
\hline $\begin{array}{l}\text { Intento comprender a todas las personas, incluso si no me } \\
\text { agradan. (ítem 9) }\end{array}$ & $3.63(1.11)$ & -0.57 & -0.21 & 14 \\
\hline $\begin{array}{l}\text { Me cuesta respetar a las personas que tienen opiniones } \\
\text { muy distintas de las mias. (Item } 10 \text {; inverso) }\end{array}$ & $2.74(1.2)$ & 0.12 & -0.81 & 15 \\
\hline $\begin{array}{l}\text { No veo ningún problema en ser impaciente con las } \\
\text { personas que me desagradan. (Item } 11 \text {; inverso) }\end{array}$ & $2.73(1.19)$ & 11 & -0.71 & 6 \\
\hline $\begin{array}{l}\text { Ya que todos somos seres humanos, todas las personas } \\
\text { deberían ser tratadas con respeto. (́tem 12) }\end{array}$ & $4.07(1.1)$ & -0.98 & 0.15 & 3 \\
\hline
\end{tabular}

Las correlaciones de los ítems de la ERIP se reportan en la Tabla 2. Si bien la gran mayoría de las correlaciones fueron significativas, todas aparecieron en la dirección esperada: en forma inversa entre los ítems redactados en el sentido de la escala y los ítems inversos y en forma directa entre los ítems de igual dirección teórica.

\section{Propiedades Psicométricas de la ERIP}

Con el fin de evaluar las dimensiones del conjunto de ítems de la ERIP, se seleccionó de modo aleatorio a $30 \%$ de participantes de la muestra total $(\mathrm{n}=287)$. Puesto que los ítems fueron respondidos con una escala ordinal de cinco puntos, se realizó un AFE, utilizando el estimador WLSMV (Weighted Least Squares Means and Variance Adjusted), basado en el análisis de correlaciones policóricas y la rotación Geomin. Se empleó la matriz con los ítems negativos sin invertir, dado que la solución factorial fue equivalente cuando se usaron los ítems negativos, salvo por el signo de las correlaciones. Dado que la versión original del ERIP demostró contar con una estructura unidimensional, se decidió probar esta misma solución y luego compararla con una de dos factores.
Tabla 2

Intercorrelaciones entre los items de la Escala de Respeto Incondicional hacia las Personas (muestra total)

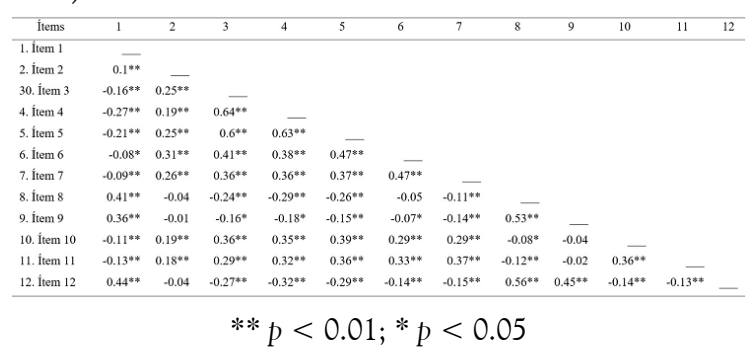

Para la solución unidimensional, los resultados de los índices de ajuste fueron: $\chi^{2}(54)=420.391$, $p<0.001 ;$ RMSEA $=0.154(0.14-0.168) ;$ CFI $=0.848 ; \mathrm{TLI}=0.814 ; \mathrm{RMR}=0.107$. Todos los índices obtenidos superaron los límites sugeridos en la literatura (i. e., RMSEA $\leq 0.06(\mathrm{Hu} \&$ Bentler, 1999); RMSEA $\leq 0.07$ (Steiger, 2007); CFI y TLI > 0.9 (Hair, Anderson, Tatham, \& Black, 2005), lo que sugiere que la ERIP no presentaría una sola dimensión. Con la finalidad de comparar estos resultados, se realizó un AFE en la misma muestra pero probando la solución de dos factores. Los valores de los índices de ajuste fueron los siguientes: $\chi^{2}(43)=145.523, p$ $<0.001$; RMSEA $=0.091(0.075-0.108)$; CFI $=0.957 ; \mathrm{TLI}=0.935 ; \mathrm{SRMR}=0.055$. Como se observa, los valores del CFI y TLI indican un buen ajuste, aunque el valor del RMSEA está por sobre los estándares recomendados. Al comparar la solución de uno y dos factores se observó un mejor ajuste del segundo modelo $\left(\Delta \chi^{2}(11)=\right.$ 197.501, $p<0.001$ ).

Seguidamente, se realizó un AFC utilizando el estimador WLSMV en el $60 \%$ de la muestra $(n=660)$. Los resultados de los índices de ajuste fueron: $\chi^{2}(53)=393.694, p<0.001$; RMSEA $=0.099$ (0.09-0.108); CFI $=0.943$; TLI $=0.929$. Nuevamente, los valores del CFI y TLI indican un buen ajuste, aunque el valor del RMSEA se encuentra ligeramente por sobre los estándares recomendados. Todas las cargas factoriales de los ítems fueron significativas y mayores a 0.3 y la correlación entre ambos factores fue -0.445 (Figura 1). Sobre la base de estos resultados, se procedió a denominar al 
Factor 1 Desprecio hacia las personas (compuesto por ocho ítems negativos) y al Factor 2 Respeto hacia las personas (compuesto por cuatro ítems positivos).

Figura 1

Modelo de dos dimensiones de la Escala de Respeto Incondicional hacia las Personas

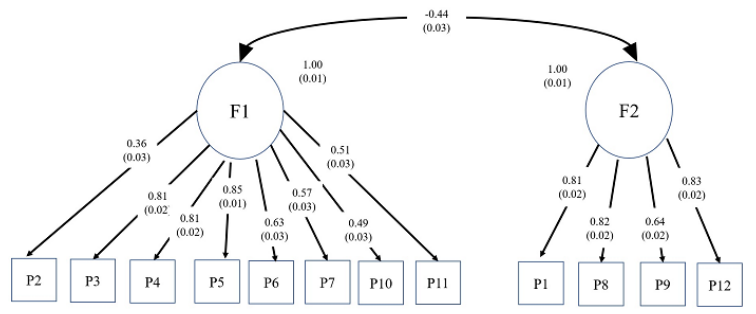

La confiabilidad de la consistencia interna de ambos factores se calculó mediante el coeficiente Omega ( $\omega$ ) de McDonald (1978). La elección de este estimador responde a la sugerencia realizada por diferentes investigadores quienes plantean que se debiera emplear este índice y no el alpha de Cronbach cuando se trabaja con ítems ordinales de respuesta, tales como las escalas tipo Likert, y estos datos son analizados con una matriz de correlaciones policóricas, como en el caso de la ERIP (Gadermann, Guhn, \& Zumbo, 2012). En consecuencia, se calculó el $\omega$ con el software libre JASP v. 0.8.3.1 (JASP, 2017). Basado en estos análisis, los factores Desprecio hacia las personas y Respeto hacia las personas obtuvieron valores de confiabilidad adecuados $(\omega=0.82$ y 0.78 , respectivamente).

Considerando la muestra total, el promedio del factor Desprecio hacia los demás fue de $2.51(D E=0.8)$ y del factor Respeto hacia los demás fue de $3.84(D E=0.84)$. Con respecto al comportamiento de los puntajes de los dos factores de la ERIP según el sexo y los distintos grupos etarios de los participantes de la muestra total, se realizó un MANOVA. Para esto, se tomó como variable de comparación el sexo, el curso y la interacción de ambas, mientras que las variables dependientes fueron los promedios de ambos factores. Luego de evaluar el cumplimiento de los supuestos de esta técnica, se detectaron diferencias significativas por sexo (F Lambda de Wilks $(2,893)=13.94, p$
$<0.001$ e eta $^{2}=0.03$ ) y por curso ( $F$ Lambda de Wilks $_{(8,1784)}=2.06, p<0.05$ eta $\left.^{2}=0.009\right)$, pero no para la interacción entre estas variables.

Las mujeres $(\mathrm{M}=3.96, \mathrm{DE}=0.81)$ mostraron mayores niveles de respeto en comparación a los hombres $(\mathrm{M}=3.75, \mathrm{DE}=0.84), \mathrm{F}(1,9.66)=$ 14.26, $\mathrm{p}<0.001$, eta ${ }^{2}=0.02$. Por su parte, los hombres $(M=2.61, D E=0.79)$ tuvieron puntajes ligeramente más altos que las mujeres $(M=2.39, D E=0.79)$ en el factor Desprecio hacia las personas, $F_{(1,9.66)}=20.31, p<0.001$, eta $^{2}=0.02$. En cuanto al análisis de los puntajes por curso, si bien solo se encontraron diferencias significativas en el factor de respeto, el tamaño del efecto fue muy bajo, $F_{(4,6.99)}=2.58, p=$ 0.04 e $^{2}{ }^{2}=0.011$, lo que indica que no hay diferencias relevantes en este constructo en los distintos niveles escolares.

Finalmente, para evaluar la validez convergente y discriminante se correlacionaron los factores de la ERIP con las cuatro subdimensiones de la escala de Dominancia Social (Dominancia, Contra la dominancia, Anti-igualitarismo y Contra el antiigualitarismo), la Conducta de Matonaje, la Convicción Moral y la Toma de Perspectiva. En la Tabla 3, se observa que todas las correlaciones fueron significativas y en la dirección esperada con cada factor de la ERIP

\section{Tabla 3}

Promedios, desviación estándar e intercorrelaciones de las otras variables del estudio con los dos factores de la ERIP

\begin{tabular}{|c|c|c|c|}
\hline & $M(D E)$ & $\begin{array}{l}\text { Desprecio hacia los } \\
\text { demás (Factor 1) }\end{array}$ & $\begin{array}{l}\text { Respeto hacia los } \\
\text { demás (Factor 2) }\end{array}$ \\
\hline 1. Dominancia & $2.16(1.06)$ & $0.45^{* *}$ & $-0.31 * *$ \\
\hline 2. Contra la dominancia & $3.78(1.07)$ & $-0.19 * *$ & $0.46^{* *}$ \\
\hline 3. Anti-igualitarismo & $2.59(1.09)$ & $0.35^{* *}$ & $-0.2 * *$ \\
\hline 4. Contra el ant-igualitarismo & $3.91(1.01)$ & $-0.19 * *$ & $0.58 * *$ \\
\hline 5. Toma de perspectiva & $3.42(0.62)$ & $-0.17 * *$ & $0.4 * *$ \\
\hline 6. Convicción Moral & $3.51(0.82)$ & $-0.09 * *$ & $0.44 * *$ \\
\hline 7. Conducta de matonaje & $1.76(0.78)$ & $0.27 * *$ & $-0.32 * *$ \\
\hline
\end{tabular}

\section{Discusión}

El objetivo central de la presente investigación fue adaptar y validar la ERIP en una muestra de escolares chilenos. Mediante análisis factoriales exploratorios y confirmatorios, se obtuvo que 
la ERIP posee una estructura bidimensional, resultado que difiere de lo encontrado en otras investigaciones (p. ej., Lalljee et al., 2007). El factor Desprecio hacia los demás aglutinó los ítems opuestos al constructo de la escala y el factor Respeto a los demás integró los ítems que aparecen en la misma dirección del constructo evaluado. La confiabilidad de ambos factores fue alta, mientras que correlaciones de nivel medio, significativas y en la dirección esperada con las otras medidas evaluadas permiten confirmar la validez convergente y discriminante de la ERIP.

La presencia de dos factores podría explicarse debido a la presencia de ítems "invertidos", lo cual ha sido observado por distintos autores (Zhang, Noor, \& Savalei, 2016). Esta estrategia presenta varias ventajas tales como corregir potenciales sesgos en las respuestas de los participantes o evitar que la persona responda de manera automática un cuestionario, sin poner atención a la diferente direccionalidad de los ítems (Weijters, Baumgartner, \& Schillewaert, 2013). Pero el uso de ítems invertidos también tiene desventajas que van desde la confusión de los participantes, debido a la dificultad de interpretar los ítems hasta la aparición de factores que los investigadores no estaban interesados en medir, como por ejemplo, el tipo de redacción de la pregunta (Van Sorenden, Sanderman, \& Coyne, 2013; Zhang \& Savalei, 2016). La aparición de dos factores en escala unidimensionales que poseen ítems invertidos puede explicarse también porque las personas responden e interpretan los ítems positivos e invertidos como constructos muy diferentes, en vez de considerarlos como extremos opuestos de un mismo constructo. En la literatura psicológica, se encuentran varios casos de instrumentos con ítems invertidos que poseen una estructura psicométrica clara y definida en sus estudios de validación inicial, pero después otros autores reportan resultados diferentes (Marsh, Scalas, \& Nagengast, 2010).

A nuestro juicio, la ERIP no cuenta con dos factores diferentes, sino que son "creados" artificialmente por el uso de ítems invertidos. Ahora bien, ipor qué esto no ocurrió en investigaciones previas? Los psicólogos transculturales han observado alteraciones en las dimensiones de instrumentos con ítems invertidos cuando son aplicados en países y lenguas diferentes de donde fueron creadas (Wong, Rindfleisch, \& Burroughs, 2003).

Con respecto a las diferencias en los puntajes de la ERIP por sexo y curso, las mujeres promediaron más alto en la dimensión de respeto, mientras que los hombres reportaron sentir más desprecio, aunque este valor estuvo por debajo del punto medio de la escala. Estos resultados no son infrecuentes, pues los hombres suelen tener más actitudes y estereotipos negativas de los grupos sociales que las mujeres (Akrami, Ekehammar, \& Anega, 2000). En cuanto a las diferencias de edad de la ERIP, si bien los promedios de los cursos fueron altos, los alumnos de octavo básico y cuarto medio tuvieron promedios ligeramente más altos que los alumnos de primero medio y tercero medio, aunque el tamaño del efecto es pequeño.

La principal fortaleza del presente estudio es la adaptación y validación de la ERIP, un instrumento que puede ser útil en el área de las relaciones intergrupales, ya que no se enfoca en la medición de actitudes hacia grupos específicos sino hacia los seres humanos en general. En ese sentido, el respeto incondicional puede ser útil para evaluar una gama de constructos que pueden aplicarse en escenarios conflictivos o cooperativos, ya sea de manera directa como las actitudes hacia los derechos humanos (McFarland, 2010) o inversa con las actitudes hacia el apoyo a la pena de muerte o la tortura (Sidanius, Mitchell, Haley, \& Navarrete, 2006).

$\mathrm{Si}$ bien se trabajó con un número relativamente grande de participantes, una limitación de este estudio fue no haber empleado una muestra probabilística, lo que impide que estos resultados puedan ser generalizables a todos los escolares chilenos. Considerando el tipo de ítems sobre los que se indagó, es posible que muchas respuestas hayan estado influidas por lo correctamente establecido por la sociedad. En ese sentido, habría sido útil haber incluido una medida de deseabilidad social. 
El respeto es un concepto esencial y de dominio general, pero su estudio psicológico solo ha sido sistemático hasta hace poco. En consecuencia, son necesarias investigaciones futuras que puedan utilizar análisis estadísticos más específicos, como la teoría de la respuesta al ítem en la ERIP, para pesquisar tendencias específicas en las respuestas de los participantes.

\section{Agradecimientos}

Esta investigación fue financiada por la Comisión Nacional de Desarrollo Científico y Tecnológico de Chile (CONICYT) a través del proyecto FONDECYT № 1151360, otorgado al primer autor.

\section{Referencias}

Akrami, N., Ekehammar, B., \& Anega, T. (2000). Classical and modern racial prejudice: A study of attitudes toward immigrants in Sweden. European Journal of Social Psychology, 30(4), 521-532. https:// doi.org/10.1002/1099-0992(200007/08)30:4 $<521:: A I D a E J S P 5>3.0 . C O ; 2-\mathrm{N}$

Blackwood, L., Hopkins, N., \& Reicher, S. (2013). Turning the analytic gaze on "us" the role of authorities in the alienation of minorities. European Psychologist, 18(4), 245-252. https://doi.org/10.1027/1016-904 0/a000151

Blader, S. L., \& Tyler, T. R. (2009). Testing and extending the group engagement model: Linkages between social identity, procedural justice, economic outcomes, and extrarole behavior. Journal of Applied psychology, 94(2), 445-464. https://doi.org/ 10.1037/a0013935

Darwall, S. L. (1977). Two kinds of respect. Ethics, 88(1), 36-49. https://doi.org/10.108 6/292054

Darwall, S. L. (2006). The secondperson standpoint: Morality, respect, and accountability. Cambridge MA: Harvard University Press.
Davis, M. H. (1983). Measuring individual differences in empathy: Evidence for a multidimensional approach. Journal of Personality and Social Psychology, 44(1), 113-126. https://doi.org/10.1037/0022-351 4.44.1.113

De Cremer, D., \& Mulder, L. B. (2007). A passion for respect: On understanding the role of human needs and morality. Gruppendynamik und Organisationsberatung [Group Dynamics and Organization Consulting], 38(4), 439-449. https://doi.or $\mathrm{g} / 10.1007 / \mathrm{s} 11612-007-0036-1$

De Cremer, D., \& Tyler, T. R. (2005). Am I respected or not?: Inclusion and reputation as issues in group membership. Social Justice Research, 18(2), 121-153. https://doi.org/10 $.1007 / \mathrm{s} 11211-005-7366-3$

Dillon, R. S. (2007). Respect: A philosophical perspective. Gruppendynamik und Organisationsberatung [Group Dynamics and Organization Consulting], 38(2), 201-212. https://doi.org/10.1007/s11 612-007-0016-5

Espelage, D. L., \& Holt, M. K. (2001). Bullying and victimization during early adolescence: Peer influences and psychosocial correlates. Journal of Emotional Abuse, 2(2-3), 123-142. https://doi.org/10.1300/J135v02n02_08

Fiske, S. T., Cuddy, A. J., Glick, P., \& $\mathrm{Xu}$, J. (2002). A model of (often mixed) stereotype content: Competence and warmth respectively follow from perceived status and competition. Journal of Personality and Social Psychology, 82(6), 878-902. https://doi.org/10.1037/0022-351 4.82.6.878

Janoff-Bulman, R., \& Werther, A. (2008). The social psychology of respect: Implications for delegitimization and reconciliation. En A. Nadler, T. Malloy \& J. D. Fisher (Eds.), Social psychology of inter-group reconciliation: From violent conflict to peaceful co-existence (pp. 145-171). Cambridge: Cambridge University Press.

Gadermann, A. M., Guhn, M., \& Zumbo, B. D. (2012). Estimating ordinal reliability for Likert-type and ordinal item response 
data: A conceptual, empirical, and practical guide. Practical Assessment, Research $\mathcal{E}$ Evaluation, 17(3), 1-13. (N. ${ }^{\circ}$ de servicio de reproducción de documentos ERIC EJ977577)

Gravetter, F. J., \& Wallnau, L. B. (2014). Essentials of statistics for the behavioral sciences (8.a ed.). Belmont, CA: Cengage Learning.

Haidt, J., \& Kesebir, S. (2010). Morality. En S. Fiske, D. Gilbert \& G. Lindzey (Eds.), Handbook of social psychology (Vol. 1, pp. 797-832). Hobeken, NJ: Wiley.

Hair, J., Anderson, R., Tatham, R., \& Black, W. (2005). Análisis multivariante. Madrid: Pearson Educación, S. A.

Ho, A. K., Sidanius, J., Kteily, N., SheehySkeffington, J., Pratto, F., Henkel, K. E., ... \& Stewart, A. L. (2015). The nature of social dominance orientation: Theorizing and measuring preferences for intergroup inequality using the new $\mathrm{SDO}_{7}$ Scale. Journal of Personality and Social Psychology, 109(6), 1003-1028. https://doi.org/10.1037/pspi00 00033

Hu, L. T., \& Bentler, P. M. (1999). Cutoff criteria for fit indexes in covariance structure analysis: Conventional criteria versus new alternatives. Structural Equation Modeling: A Multidisciplinary Journal, 6(1), 1-55. http s://doi.org/10.1080/10705519909540118

Huo, Y. J., \& Molina, L. E. (2006). Is pluralism a viable model of diversity? The benefits and limits of subgroup respect. Group Processes $\mathcal{E}$ Intergroup Relations, 9(3), 359-376. https:// doi.org/10.1177/1368430206064639

Janoff-Bulman, R., \& Werther, A. (2008). The social psychology of respect: Implications for delegitimization and reconciliation. En A. Nadler, T. Malloy \& J. D. Fisher (Eds.), Social psychology of inter-group reconciliation: From violent conflict to peaceful co-existence (pp. 145-171). Cambridge: Cambridge University Press.

JASP Team (2018). JASP (Version 0.9) [Computer software]. Amsterdam, NLD: University of Amsterdam.
Kant, I. (1995). Fundamentación de la metafísica de las costumbres. Crítica de la razón práctica. La paz perpetua. México: Porrúa. (Obra originalmente publicada en 1785)

Lægard, S. (2010). Recognition and toleration: Conflicting approaches to diversity in education? En M. Sardoč (Ed.), Toleration, respect and recognition in education (pp. 17-32). Kuala Lumpur, MY: WileyBlackwell.

Laham, S. M., Tam, T., Lalljee, M., Hewstone, M., $\&$ Voci, A. (2010). Respect for persons in the intergroup context: Self-other overlap and intergroup emotions as mediators of the impact of respect on action tendencies. Group Processes $\mathscr{E}$ Intergroup Relations, 13(3), 301-317. https://doi.org/10.1177/13 68430209344606

Lalljee, M., Laham, S., \& Tam, T. (2007). Unconditional respect for persons: A social psychological analysis. Gruppendynamik und Organisationsberatung [Group Dynamics and Organization Consulting], 38(4), 451-464. https://doi.org/10.1007/s11612-0 07-0037-0

Lalljee, M., Tam, T., Hewstone, M., Laham, S. M., \& Lee, J. (2009). Unconditional respect for persons and the prediction of intergroup action tendencies. European Journal of Social Psychology, 39, 666-683. ht tps://doi.org/10.1002/ejsp.564

Lawrence-Lightfoot, S. (2000). Respect: An exploration. Cambridge: Perseus.

Leary, J. D., Brennan, E. M., \& Briggs, H. E. (2005). The African American Adolescent Respect Scale: A measure of a prosocial attitude. Research on Social Work Practice, 15(6), 462-469. https://doi.org/10.1177/10 49731505277717

Licata, L., Sánchez-Masas, M., \& Green E. G. T. (2011). Identity, immigration, and prejudice in Europe: A recognition approach. En S. J. Schwartz, K. Luyckx \& V. L. Vignoles (Eds.), Handbook of identity theory and research (Vol. 1., pp. 895-916). Nueva York: Springer.

Marsh, H. W., Scalas, L. F., \& Nagengast, B. (2010). Longitudinal tests of competing 
David Sirlopú, Claudia Pérez-Salas, Verónica Villarroel, et al.

factor structures for the Rosenberg SelfEsteem Scale: Traits, ephemeral artifacts, and stable response styles. Psychological Assessment, 22(2), 366-381. https://doi.org/ 10.1037/a0019225

McDonald, R. P. (1978). Generalizability in factorable domains: "Domain validity and generalizability". Educational and Psychological Measurement, 38(1), 75-79. htt ps://doi.org/10.1177/001316447803800111

McFarland, S. (2010). Personality and support for universal human rights: A review and test of a structural model. Journal of Personality, 78(6), 1735-1763. https://doi.org/10.1111/j $.1467-6494.2010 .00668 . x$

Mestre Escrivá, V., Frías Navarro, M. D., \& Samper García, P. (2004). La medida de la empatía: Análisis del Interpersonal Reactivity Index. Psicothema, 16(2), 255-260. Recuperado de http://www .psicothema.com/pdf/1191.pdf

Muñiz, J., Elosua, P., \& Hambleton, R. K. (2013). Directrices para la traducción y adaptación de los tests: segunda edición. Psicothema, 25(2), 151-157. https://doi.org/10.7334/psi cothema2013.24

Renger, D., Mommert, A., Renger, S., \& Simon, B. (2016). When less equal is less human: Intragroup (dis) respect and the experience of being human. The Journal of Social Psychology, 156(5), 553-563. https://doi.org $/ 10.1080 / 00224545.2015 .1135865$

Renger, D., \& Simon, B. (2011). Social recognition as an equal: The role of equality-based respect in group life. European Journal of Social Psychology, 41 (4), 501-507. https://doi.org/10.1002/ejsp.814

Schirmer, W., Weidenstedt, L., \& Reich, W. (2012). From tolerance to respect in interethnic contexts. Journal of Ethnic and Migration Studies, 38(7), 1049-1065. https:/ /doi.org/10.1080/1369183X.2012.681448

Sidanius, J., Mitchell, M., Haley, H., \& Navarrete, C. D. (2006). Support for harsh criminal sanctions and criminal justice beliefs: A social dominance perspective. Social Justice Research, 19, 433-449. https:// doi.org/10.1007/s11211-006-0026-4
Simon, B. (2007). Respect, equality, and power: A social psychological perspective. Gruppendynamik und Organisationsberatung [Group Dynamics and Organization Consulting], 38(3), 309-326. https://doi.or $\mathrm{g} / 10.1007 / \mathrm{s} 11612-007-0027-2$

Simon, B., \& Grabow, H. (2014). To be respected and to respect: The challenge of mutual respect in intergroup relations. British Journal of Social Psychology, 53(1), 39-53. https://doi.org/10.1111/bjso.12019

Simon, B., Mommert, A., \& Renger, D. (2015). Reaching across group boundaries: Respect from outgroup members facilitates recategorization as a common group. British Journal of Social Psychology, 54, 616-628. ht tps://doi.org/10.1111/bjso.12112

Steiger, J. H. (2007). Understanding the limitations of global fit assessment in structural equation modeling. Personality and Individual Differences, 42 (5), 893-898. h ttps://doi.org/10.1016/j.paid.2006.09.017

Tyler, T. R., \& Lind, E. A. (1992). A relational model of authority in groups. Advances in Experimental Social Psychology, 25, 115-191. https://doi.org/10.1016/S0065 -2601(08)60283-X

Van Quaquebeke, N., Henrich, D. C., \& Eckloff, T. (2007). "It's not tolerance I'm asking for, it's respect!" A conceptual framework to differentiate between tolerance, acceptance and (two types of) respect. Gruppendynamik und Organisationsberatung [Group Dynamics and Organization Consulting], 38(2), 185-200. https://doi.org/10.1007/s11612-0 07-0015-6

Van Sorenden, E., Sanderman, R., \& Coyne, J. C. (2013). Ineffectiveness of reverse wording of questionnaire items: Let's learn from cows in the rain. PLoS One, 8(7), e68967. https://doi.org/10.1371/journal.po ne.0068967

van Zomeren, M., Postmes, T., \& Spears, R. (2012). On conviction's collective consequences: Integrating moral conviction with the social identity model of collective action. British Journal of Social 
Psychology, 51(1), 52-71. https://doi.org/10. 1111/j.2044-8309.2010.02000.x

Weijters, B., Baumgartner, H., \& Schillewaert, N. (2013). Reversed item bias: An integrative model. Psychological Methods, 18(3), 320-324. https://doi.org/10.1037/a0 032121

Wong, N., Rindfleisch, A., \& Burroughs, J. E. (2003). Do reverse-worded items confound measures in cross-cultural consumer research? The case of the material values scale. Journal of Consumer Research, 30(1), 72-91. http://dx.doi.org/10.1086/374697

Zhang, X., Noor, R., \& Savalei, V. (2016). Examining the effect of reverse worded items on the factor structure of the need for cognition scale. PloS one, 11(6), e0157795. https://doi.org/10.1371/journal. pone.0157795

Zhang, X., \& Savalei, V. (2016). Improving the factor structure of psychological scales: The Expanded format as an alternative to the Likert scale format. Educational and Psychological Measurement, 76(3), 357-386. https://doi.org/10.1177/00 13164415596421

\section{Notas}

* Artículo de investigación. 